\title{
Multiscale Entropy Analysis of Gravitational Waves
}

\author{
Mohsen Javaherian $\mathbb{D}^{1}$ and Saeid Mollaei $\mathbb{D}^{2}$ \\ ${ }^{1}$ Research Institute for Astronomy and Astrophysics of Maragha (RIAAM), University of Maragheh, P.O. Box: 55136-553, \\ Maragheh, Iran \\ ${ }^{2}$ Department of Physics, University of Zanjan, P.O. Box: 45196-313, Zanjan, Iran
}

Correspondence should be addressed to Mohsen Javaherian; m_javaherian@znu.ac.ir

Received 4 December 2020; Revised 17 January 2021; Accepted 22 February 2021; Published 8 March 2021

Academic Editor: Enrico Lunghi

Copyright ( 92021 Mohsen Javaherian and Saeid Mollaei. This is an open access article distributed under the Creative Commons Attribution License, which permits unrestricted use, distribution, and reproduction in any medium, provided the original work is properly cited. The publication of this article was funded by SCOAP ${ }^{3}$

\begin{abstract}
The first gravitational-wave (GW) signal was detected in the year 2015 indicating tiny distortions of spacetime caused by accelerated masses. We focused on the GW signals consisting of a peak GW strain of $1.0 \times 10^{-21}$ that shows merging pairs of large masses. We applied the generalized entropy known as multiscale entropy to the GW interval time series recorded by different observatories (H1, L1, and V1). This enables us to investigate the behavior of entropies on different scales as a method of studying complexity and organization. We found that the entropies of GW interval data with similar physical properties make the identical manner in different scales. Moreover, the results reveal that the signals collected by each observatory have approximately a similar trend in the multiscale analysis results. According to our findings, although different signals have different values for short-range correlations, the long-range correlations are not noticeable in most of them.
\end{abstract}

\section{Introduction}

Gravitational-wave (GW) signals, firstly predicted by Albert Einstein in 1916, are known as transverse waves of spatial strain with very small amplitudes which travel at the speed of light $[1,2]$. After linearizing weak-field equations, Sitzungsber found a solution for the field equations [3], and then, Kerr generalized the solution for the rotating black holes [4]. Lots of theoretical works in the purification of analytical studies of relativistic two-body dynamics were done [5, 6]. Moreover, since the signals of GW merger are accompanied with electromagnetic emission as a gamma-ray burst [7-9], the field of numerical-relativity simulations progressed, and in the later step, it led to advances in GW modeling of two massive binary mergers (e.g., see [10-14]).

In the last decade, the GW signals are detected by the networks of the Virgo [15] and LIGO [16] interferometers. The first observation of GWs, which was appeared as a transient GW signal, indicated a binary black hole merger [17]. For other detections of GW mergers, the reader can refer to $[18,19]$ and references therein. Since the GW sources can be categorized in one of the three classes of transient and bursts, periodic or continuous wave, and stochastic, the type of method for analyzing GW data seems to be important [20]. GWs are very weak signals of order $10^{-21}$ or even less that travel in spacetime containing noises of order $10^{-18}$. So, it is not possible to study this kind of signal using methods that are prevalent in statistics.

Using the Hurst exponent is one of the proposed ways to discriminate stochastic (irregular) time series and signals with long-range interaction within system components (self-affinity) which was firstly proposed by Hurst in 1951 $[21,22]$. In this approach, for a time series $\left[y_{t}\right]$, a selfaffinity of different parts of a signal can be explained by $y_{\alpha t}$ $=\alpha^{-H} y_{t}$, wherein $\alpha$ and $H$ are a positive coefficient and the Hurst exponent, respectively (e.g., see [23]). The range of $(0.5,1)$ for the Hurst exponent determines that there would be long-term memory in the system of interest. Among lots of methods for estimation of the Hurst exponent such as the analyses of detrended fluctuation [24], rescale range (e.g., [25]), and wavelet method [26], using the concept of entropy enables us to issue solid results about the complexity of a system and give a quantitative interpretation of longterm memory in the system [27]. The multiscale entropy 
TABle 1: The list of GW data.

\begin{tabular}{|c|c|c|c|c|c|c|}
\hline Name & Version & $\begin{array}{c}\text { Mass } 1 \\
\left(\mathrm{M}_{\odot}\right)\end{array}$ & Mass $2\left(\mathrm{M}_{\odot}\right)$ & Network SNR & Distance (Mpc) & Strains \\
\hline GW150914 & v3 & $35.6_{-3.1}^{+4.7}$ & $30.6_{-4.4}^{+3.0}$ & 24.4 & $440_{-170}^{+150}$ & H1 \& L1 \\
\hline GW151012 & v3 & $23.2_{-5.5}^{+14.9}$ & $13.6_{-4.8}^{+4.1}$ & 10.0 & $1080_{-490}^{+550}$ & H1 \& L1 \\
\hline GW151226 & v2 & $13.7_{-3.2}^{+8.8}$ & $7.7_{-2.5}^{+2.2}$ & 13.1 & $450_{-190}^{+180}$ & H1 \& L1 \\
\hline GW170104 & v2 & $30.8_{-5.6}^{+7.3}$ & $20.0_{-4.6}^{+4.9}$ & 13 & $990_{-430}^{+440}$ & H1 \& L1 \\
\hline GW170608 & v3 & $11.0_{-1.7}^{+5.5}$ & $7.6_{-2.2}^{+1.4}$ & 14.9 & $320_{-110}^{+120}$ & H1 \& L1 \\
\hline GW170729 & v1 & $50.2_{-10.2}^{+16.2}$ & $34.0_{-10.1}^{+9.1}$ & 10.2 & $2840_{-1360}^{+1400}$ & H1, L1 \& V1 \\
\hline GW170809 & $\mathrm{v} 1$ & $35.0_{-5.9}^{+8.3}$ & $23.8_{-5.2}^{+5.1}$ & 12.4 & $1030_{-390}^{+320}$ & $\mathrm{H} 1, \mathrm{~L} 1 \& \mathrm{~V} 1$ \\
\hline GW170814 & v3 & $30.6_{-3.0}^{+5.6}$ & $25.2_{-4.0}^{+2.8}$ & 15.9 & $600_{-220}^{+150}$ & $\mathrm{H} 1, \mathrm{~L} 1 \& \mathrm{~V} 1$ \\
\hline GW170817 & v3 & $1.46_{-0.10}^{+0.12}$ & $\begin{array}{l}1.27_{-0.09}^{+0.09} \\
\text { a }\end{array}$ & 33 & $40_{-15}^{+7}$ & $\mathrm{H} 1, \mathrm{~L} 1 \& \mathrm{~V} 1$ \\
\hline GW170818 & $\mathrm{v} 1$ & $35.4_{-4.7}^{+7.5}$ & $26.7_{-5.2}^{+4.3}$ & 11.3 & $1060_{-380}^{+420}$ & $\mathrm{H} 1, \mathrm{~L} 1 \& \mathrm{~V} 1$ \\
\hline GW170823 & v1 & $39.5_{-6.7}^{+11.2}$ & $29.0_{-7.8}^{+6.7}$ & 11.5 & $1940_{-900}^{+970}$ & H1 \& L1 \\
\hline GW190412 & v2 & $30.1_{-5.3}^{+4.6}$ & $8.3_{-0.9}^{+1.6}$ & $19.0_{-0.3}^{+0.2}$ & $740_{-160}^{+130}$ & $\mathrm{H} 1, \mathrm{~L} 1 \& \mathrm{~V} 1$ \\
\hline GW190425 & $\mathrm{v} 1$ & $1.74_{-0.09}^{+0.17}$ & $1.56_{-0.14}^{+0.08}$ & $12.46_{-0.43}^{+0.29}$ & $159_{-72}^{+69}$ & $\mathrm{~L} 1 \& \mathrm{~V} 1$ \\
\hline GW190521 & v2 & $85_{-14}^{+21}$ & $66_{-18}^{+17}$ & $14.6_{-0.4}^{+0.4}$ & $5300_{-2600}^{+2400}$ & $\mathrm{H} 1, \mathrm{~L} 1 \& \mathrm{~V} 1$ \\
\hline GW190814 & $\mathrm{v} 1$ & $23.2_{-1.0}^{+1.1}$ & $2.59_{-0.09}^{+0.08}$ & $25.0_{-0.2}^{+0.1}$ & $241_{-45}^{+41}$ & $\mathrm{H} 1, \mathrm{~L} 1 \& \mathrm{~V} 1$ \\
\hline
\end{tabular}

(MSE) is firstly introduced in a paper for analyzing the complexity of physiologic time series [28], and then, it was broadly used in biological signals such as characterizing the complexity of human heartbeat $[29,30]$. For a review of other improvements about the MSE estimation, the reader can refer to [31].

Here, we applied the MSE to GW binary mergers to investigate the entropy level of signals in different scales. To do this, we employed the GW signals of events detected by different observatories. It may help to characterize the GW signals. This paper is organized as follows: the employed data sets are described in Section 2. Section 3 is devoted to briefly explaining the method. The results are discussed in Section 4. The concluding remarks are given in Section 5.

\section{Description of Data Sets}

The networks of the Virgo interferometers in Cascina, Italy, [15] and the Laser Interferometer Gravitational-wave Observatory [LIGO: [16] including two branches in Hanford, Washington, USA (LHO), and Livingstone, Louisiana, USA (LLO), with the arms of $4 \mathrm{~km}$ record the received GW signals]. Recorded data are strains $(\Delta L / L)$ of the arms, caused by gravitational waves; so, they are dimensionless. The data published by the Virgo observatory is labeled "V1," and the two branches of LIGO detectors are known as "H1" and "L1," respectively. We employed the GW time series (https://www.gw-openscience.org/data/) with the sampling rate of $16 \mathrm{KHz}$ during the period of 32 seconds (the time of recording data). The employed data sets are categorized into two groups. In the first group, the physical properties of triggered events such as masses and distances of binaries have
TABLE 2: The list of GWTC-1-marginal data.

\begin{tabular}{|c|c|c|c|c|c|c|}
\hline Name & Version & $\begin{array}{c}\text { Mass } 1 \\
\left(\mathrm{M}_{\odot}\right)\end{array}$ & $\begin{array}{c}\text { Mass } 2 \\
\left(\mathrm{M}_{\odot}\right)\end{array}$ & $\begin{array}{c}\text { Network } \\
\text { SNR }\end{array}$ & $\begin{array}{c}\text { Distance } \\
(\mathrm{Mpc})\end{array}$ & Strains \\
\hline 170208 & v1 & - & - & 10.0 & - & $\begin{array}{c}\text { H1 \& } \\
\text { L1 }\end{array}$ \\
\hline 170219 & $\mathrm{v} 1$ & - & - & 9.6 & - & $\begin{array}{c}\text { H1 \& } \\
\text { L1 }\end{array}$ \\
\hline 170405 & v1 & - & - & 9.3 & - & $\begin{array}{c}\text { H1 \& } \\
\text { L1 }\end{array}$ \\
\hline 170412 & v1 & - & - & 9.7 & - & $\begin{array}{c}\text { H1 \& } \\
\text { L1 }\end{array}$ \\
\hline 170423 & $\mathrm{v} 1$ & - & - & 8.9 & - & $\begin{array}{c}\text { H1 \& } \\
\text { L1 }\end{array}$ \\
\hline
\end{tabular}

been found. We selected all confirmed GW data consisting of 15 time series of compact binary mergers recorded by different observatories. The second group is marginal triggers observed by LIGO and Virgo discovered by the advanced interferometric GW detector network. This network can detect frequencies in the range of $15 \mathrm{~Hz}$ up to a few kilohertz belonging to inspiral, merger, and ringdown GW signals of compact binary mergers (for more information about this type of data set and some technical details, the reader can refer to [32]). They are listed in the GW transient catalog and are known as "GWTC-1-marginal." We selected 5 times series of this type of GW data. Details of the first and the second groups of the GW data are given in Tables 1 and 2, respectively (also, the extended reviews about the Advanced LIGO and Advanced Virgo data sets are given in [33]). Both groups of data sets are named by the date of observations with a difference that the names of the first group have the 
prefix "GW." For instance, the series "GW190425" belongs to the first group and was recorded on 25 April 2019.

For a given data set $B_{H}(t)$ (a time series with a Hurst exponent of $H$ ), we can produce a data set from its increments as $G_{H}(t)=\Delta B_{H}(t)=B_{H}(t+\Delta t)-B_{H}(t)$, which is known as interval data set. Our measurements show that the $G_{H}(t)$ constructed from $\mathrm{GW}$ time series (listed in Tables 1 and 2) has Gaussian distribution. Also, their Hurst exponents are ranged from 0.61 to 0.89 (extracted by the $\mathrm{R} / \mathrm{S}$ method) led to categorize GW interval signals in the class of the fractional Gaussian noises. We apply multiscale entropy to GW interval data sets as explained in Section 3.

\section{Multiscale Entropy Analysis}

The time series of a system can be influenced by noises rooted in the interaction between the system and its environment. Inducing noise can accumulate short-range correlations in time series that may lead to a nonoriginal long-range effect. So, reducing the effect of undesirable noises and shortrange correlations from time series seems to be essential. This task is accomplished by using the coarse-graining procedure. To do this, the time series of length $N$ with data points $y_{1}$, $y_{2}, \cdots, y_{N}$ is partitioned into nonoverlapping windows with the same length $\lambda$. Averaging on data points over each window provides the coarse-grained signal as the following form

$$
y_{p}^{(\lambda)}=\frac{1}{\lambda} \Sigma_{q=1}^{\lambda} y_{(p-1) \lambda+q} .
$$

The new sequence with data points $\left(y_{1}^{(\lambda)}, \cdots, y_{N / \lambda}^{(\lambda)}\right)$ is obtained, wherein $\lambda$ is known as scale factor. The late signal can be determined by the $m$-dimensional vectors as follows:

$$
Y_{m}^{(\lambda)}(p)=\left(y_{p}^{\lambda}, \cdots, y_{p+m-1}^{\lambda}\right) .
$$

In this step, the number of vector pairs with distances less than $r$ is counted. This set of pairs is denoted by $n_{m}(r, \lambda)$. The task of finding the pairs set is repeated for the $(m+1)$ -dimensional vector pairs $n_{m+1}(r, \lambda)$. The sample entropy is given by

$$
S_{E}(m, r, \lambda)=-\log \left(\frac{n_{m+1}(r, \lambda)}{n_{m}(r, \lambda)}\right)
$$

Since $n_{m+1}(r, \lambda) \leq n_{m}(r, \lambda)$, then $S_{E} \geq 0$. The diagram of sample entropy versus scale factor can be representative of the range of correlations. The whole process is named the multiscale entropy (MSE) analysis [27].

\section{Results and Discussions}

We plotted the sample entropy of GW interval data for different scale factors. To do this, $15 \mathrm{GW}$ signals received from binary mergers with some discovered physical properties and 5 marginal triggers (GWTC-1-marginal data) with indeterminate parameters of sources were chosen to generate interval data. The results of MSE analysis for binary mergers
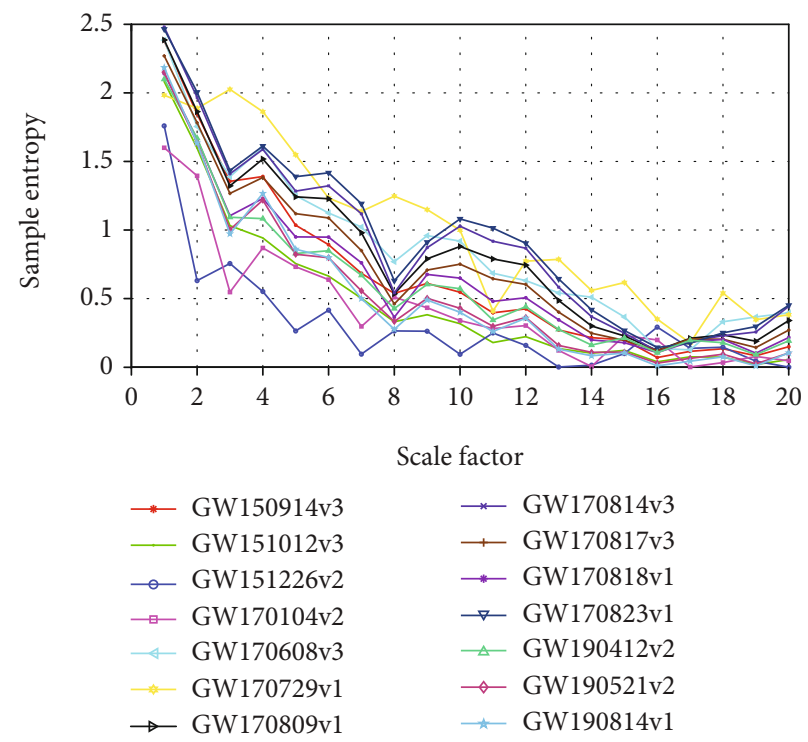

FIgURe 1: MSE analysis of the GW interval data recorded by $\mathrm{H} 1$ observatory.

recorded by observatories $\mathrm{H} 1, \mathrm{~L} 1$, and $\mathrm{V} 1$ are shown in Figures $1-3$, respectively.

As we see in Figure 1, approximately all graphs have analogous trends, and despite consecutive peaks and valleys, they all exhibit a decrement of sample entropy toward values below 0.5 . In scale 8 , a valley can be seen for most of the graphs except for four of them. Also, there is a progressing convergence reaching its maximum value in the range $[16$, 17] which is the other noticeable case in Figure 1. In Figure 2, the decreasing trend is repeated with a less obvious convergence between graphs. One can see that the MSE analysis for the last six recorded data has very close and similar trends. There is a valley in scale 16 for all graphs, and then, the first four recorded data begin to show a divergent manner gradually. In Figure 3, alongside the decrement of all trends toward zero, a duality can be seen among the graphs. Both groups of the graphs show consecutive peaks and valleys, but it is obvious that the entropy behavior of the last four recorded data is different from the others' trend, and the trends of the two groups of graphs follow the decline trends completely out of phase after scale 11 .

In the same manner, as explained above, the results for interval data generated from marginal triggers recorded by observatories $\mathrm{H} 1$ and L1 are displayed in Figures 4 and 5, respectively. For the two last interval data generated from GWTC-1-marginal H1 data discovered in April 2017, it is seen that the sample entropies show the same behavior while the others' trends exhibit gradual declines through all scales. The results for GWTC-1-marginal L1 data (except for the signal taken on 8 February 2017 that is the first recoded data) show similar falling trends in MSE graphs with fewer peaks and valleys in comparing with the results of GWTC-1marginal H1. For all the graphs of $15 \mathrm{GW}$ signals and 5 marginal triggers that monotonically decrease, the best model can be obtained by fitting a $q$-exponential function, $f(x)=$ $A[1-B(q-1) x]^{1 / 1-q}$, wherein $A, B$, and $q$ are constant values. 


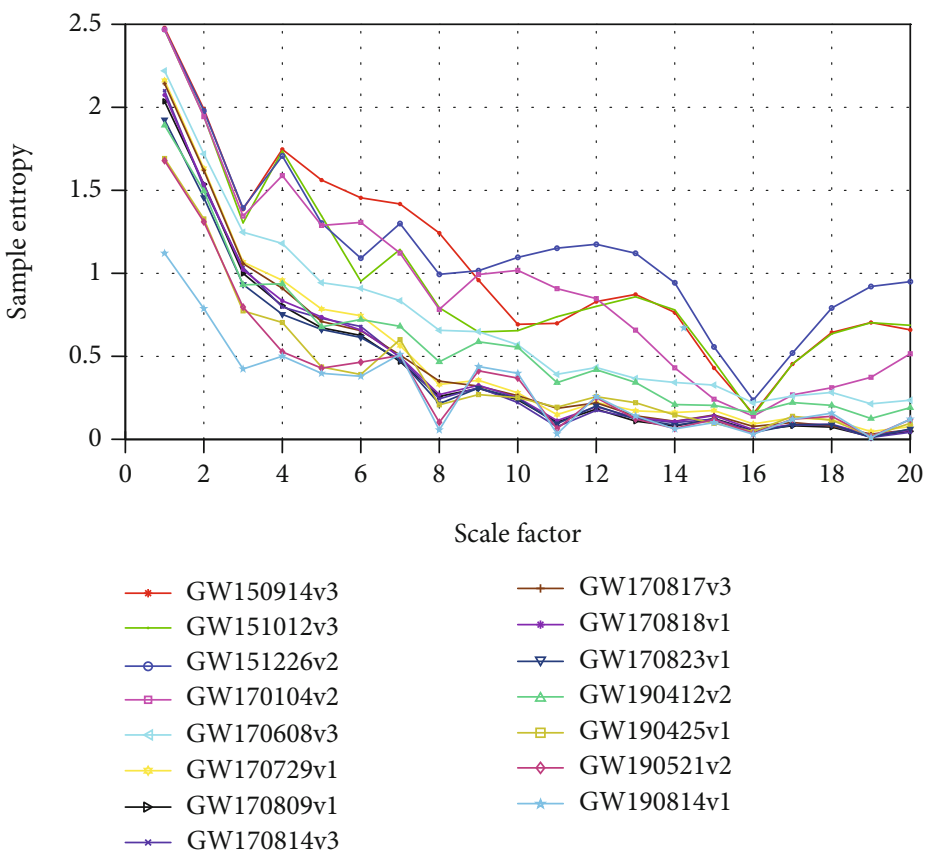

FIGURE 2: MSE analysis of the GW interval data recorded by L1 observatory.

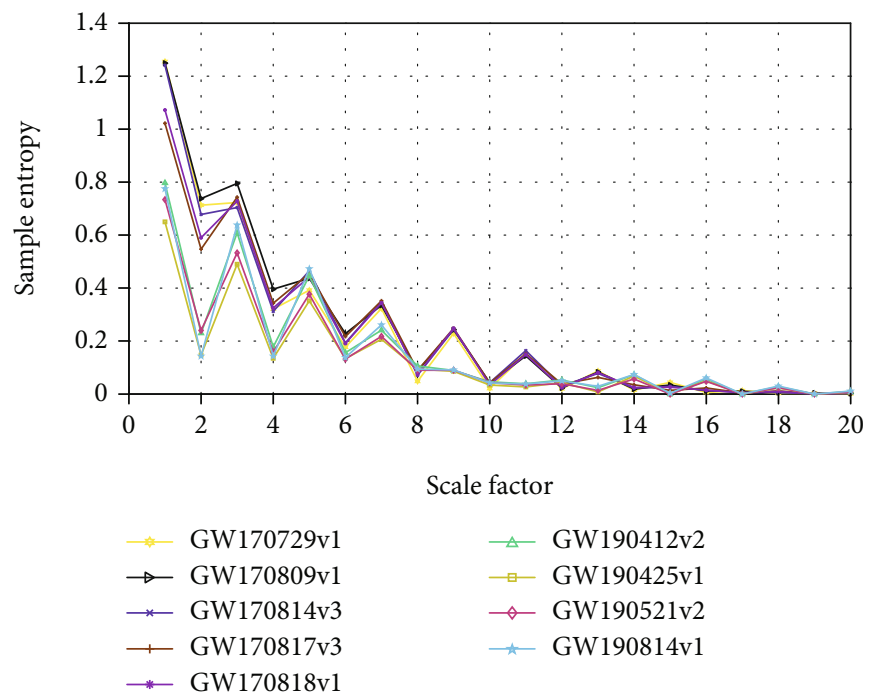

Figure 3: MSE analysis of the GW interval data recorded by V1 observatory.

To know whether there is any relation between the physical parameters of GW data and behavior of sample entropy for their corresponding interval data in different scales, we focused on masses, distances, and network SNR of binary mergers. First of all, we compared the entropies of two GW interval data with comparable characteristics in different scales. As we see in Table 1, both the GW170809 (with mass $1 \approx 35.0 \mathrm{M}_{\odot}$, mass $2 \approx 23.8 \mathrm{M}_{\odot}$, distance $\approx 1030 \mathrm{Mpc}$, and network SNR $\approx 12.4$ ) and the GW170818 (with mass 1 $\approx 35.4 \mathrm{M}_{\odot}$, mass $2 \approx 26.7 \mathrm{M}_{\odot}$, distance $\approx 1060 \mathrm{Mpc}$, and network SNR $\approx 11.3$ ) have approximately the analogous properties. Their entropy trends are very similar in different scales recorded by $\mathrm{H} 1$ (Figure 1), L1 (Figure 2), and V1 (Figure 3). Then, we focused on two GW time series with dif- ferent physical properties. As it is seen in Table 1, the mergers of GW190521 data are more massive (mass $1 \approx 85 \mathrm{M}_{\odot}$ and mass $266 \mathrm{M}_{\odot}$ ) than the others, and also, the distance of the mergers from detectors is significant $(\approx 5300 \mathrm{Mpc})$. On the other hand, the GW170817 mergers have the lowest masses (mass $1 \approx 1.46 \mathrm{M}_{\odot}$ and mass $2 \approx 1.27 \mathrm{M}_{\odot}$ ) and are placed in a shorter distance than the other mergers $(\approx 40 \mathrm{Mpc})$. Let us make a comparison between the entropy diagrams of mergers of the GW190521 with SNR $\approx 14.6$ and the GW170817 with SNR $\approx 33$. A closer look at results extracted from data recorded by $\mathrm{H} 1$ (Figure 1) shows that both approximately have similar trends with in an in-phase manner. However, the values of sample entropy for GW170817 are higher than that of GW190521 in all scales. In Figure 2, we 


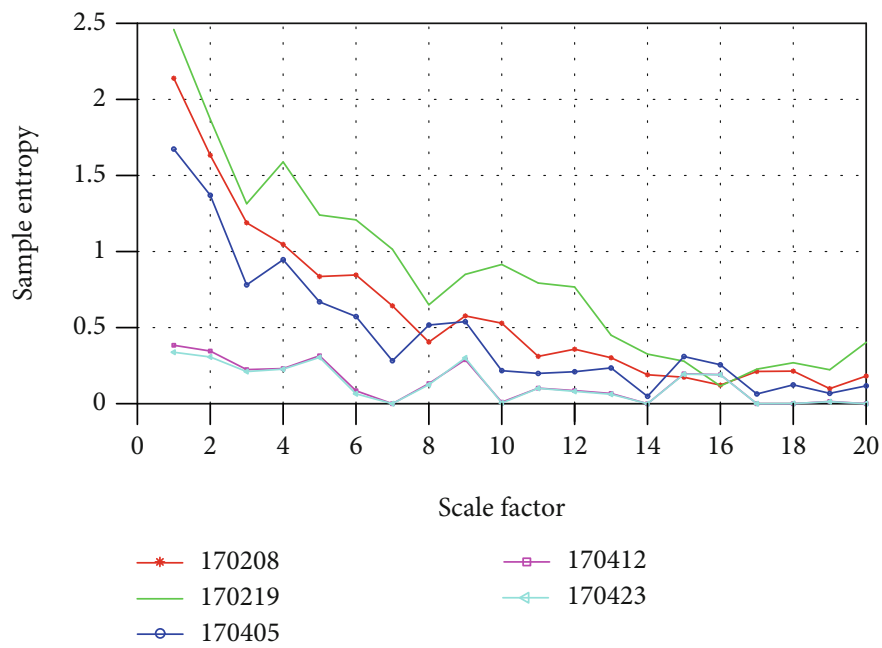

FIGURE 4: MSE analysis of the GWTC-1-marginal interval data recorded by $\mathrm{H} 1$ observatory.

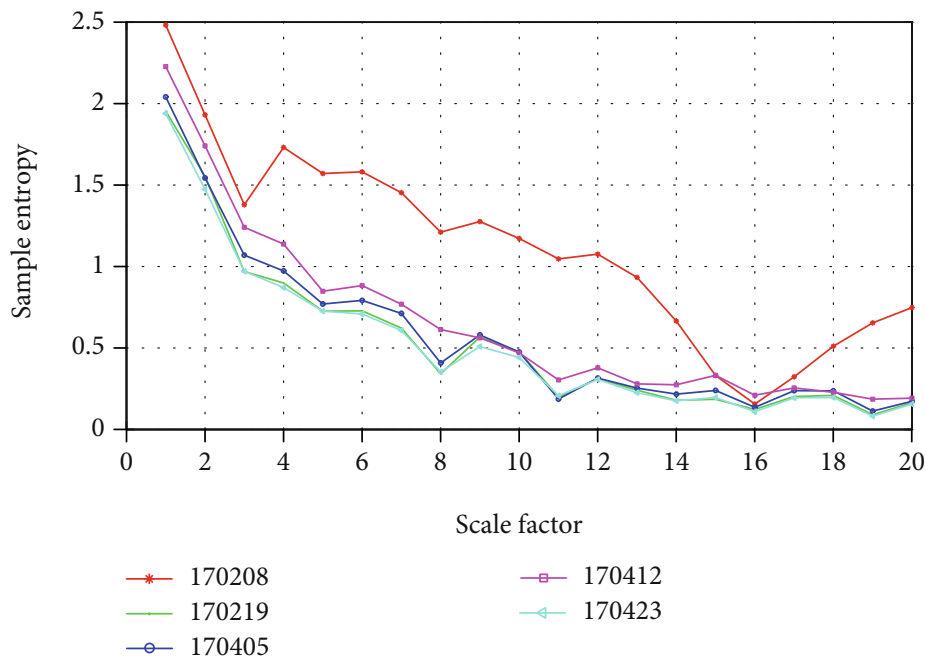

FIGURE 5: MSE analysis of the GWTC-1-marginal interval data recorded by L1 observatory.

see the same trends for both data. The MSE diagram of GW190521 has more fluctuations than that of obtained for GW170817, and it takes the greater values at some scales. We see in Figure 3 that rises and falls of both data are very similar up to scale 10; after that, although both approximately have the analogous slopes, the fluctuation of GW190521 data is more. In the GWTC-1-marginal data set, the only recorded property is network SNR that is approximately comparable for 170219 (network SNR ₹9.6) and 170412 (network SNR $\approx 9.7$ ) signals. The MSE diagrams of the 170219 and 170412 interval data sets recorded by $\mathrm{H} 1$ show that their entropy behaviors are completely different. The sample entropy of 170412 data takes the value zero in some scales, while the minimum value of 170219 data $(\approx 0.21)$ is in the scale 16 . On the other hand, for the data recorded by L1, the entropy behaviors are similar. Hence, we can say that the network SNR does not play a key role in determining the entropy behaviors of the GW signals.

\section{Conclusions}

In this study, we first generated interval data sets from GW data and measured their Hurst exponent using R/S analysis. Our measurements show that the interval data have the Hurst exponents ranged from 0.61 to 0.89 . We applied MSE analysis as a method of investigating complexity and organization in time series, on two groups of interval data generated from GW data recorded by different observatories (Virgo and LIGO). We found that the GW interval signals with similar sources represent analogous behaviors in different scales. Focusing on entropy diagrams of GWTC-1marginal interval data with about the same network SNR demonstrates that the entropy behaviors of the GW interval time series cannot relate to the network SNRs of observatories. Moreover, the entropy-scale diagrams show that the entropy behaviors of the GW interval data are analogous for each observatory which may return to the characteristics 
of each observatory depended on input noises, noise reduction methods, and/or the orientation of the received GW signals. All of the multiscale entropy analysis results for corresponding interval data recorded by $\mathrm{H} 1, \mathrm{~L} 1$, and $\mathrm{V} 1$ show a decreasing trend with some degrees of convergence. The diversity of the entropy in small scales implies that the signals have different values of short-range correlations. By increasing the scale factor, the effects of short-range correlations are excluded from signals. The declining trend of entropy indicates that the long-term correlations cannot have an effective influence on the system. The observed convergence in the MSE graphs, especially during the last scale factors, can be interpreted as the existence of similarity between all the GW interval time series in lack of longrange correlations. It suggests that this type of GW signal should be categorized in the class of systems with a low level of complexity.

\section{Data Availability}

We employed the GW time series with a sampling rate of $16 \mathrm{KHz}$ during the period of 32 seconds (the time of recording data). The link of used data sets is as follows: https://www .gw-openscience.org/data/.

\section{Conflicts of Interest}

The authors declare that they have no conflicts of interest.

\section{Acknowledgments}

This research has made use of data, software, and/or web tools obtained from the Gravitational Wave Open Science Center (https://www.gw-openscience.org/), a service of LIGO Laboratory, the LIGO Scientific Collaboration, and the Virgo Collaboration. LIGO Laboratory and Advanced LIGO are funded by the United States National Science Foundation (NSF) as well as the Science and Technology Facilities Council (STFC) of the United Kingdom, the MaxPlanck-Society (MPS), and the State of Niedersachsen/Germany for support of the construction of Advanced LIGO and construction and operation of the GEO600 detector. Additional support for Advanced LIGO was provided by the Australian Research Council. Virgo is funded, through the European Gravitational Observatory (EGO), by the French Centre National de Recherche Scientifique (CNRS), the Italian Istituto Nazionale di Fisica Nucleare (INFN), and the Dutch Nikhef, with contributions by institutions from Belgium, Germany, Greece, Hungary, Ireland, Japan, Monaco, Poland, Portugal, and Spain. Also, the authors thank K. Derakhshani and Professor Amir H. Darooneh for helpful discussions.

\section{References}

[1] A. Einstein, "Erklarung der perihelionbewegung der Merkur aus der allgemeinen relativitatstheorie," Sitzungsberichte der Königlich Preussischen Akademie der Wissenschaften, vol. 47, pp. 831-839, 1915.
[2] A. Einstein, "Über gravitationswellen," Sitzungsberichte der Königlich Preussischen Akademie der Wissenschaften, vol. 1, p. 154, 1918.

[3] K. Schwarzschild, "Über das Gravitationsfeld eines Massenpunktes nach der Einsteinschen Theorie," Sitzungsberichte der Königlich Preussischen Akademie der Wissenschaften, vol. 1, p. 189, 1916.

[4] R. P. Kerr, "Gravitational field of a spinning mass as an example of algebraically special metrics," Physical Review Letters, vol. 11, no. 5, pp. 237-238, 1963.

[5] A. Buonanno and T. Damour, "Effective one-body approach to general relativistic two-body dynamics," Physical Review D, vol. 59, no. 8, article 084006, 1999.

[6] L. Blanchet, "Gravitational radiation from post-Newtonian sources and inspiralling compact binaries," Living Reviews in Relativity, vol. 17, no. 1, p. 2, 2014.

[7] R. Narayan, B. Paczynski, and T. Piran, "Gamma-ray bursts as the death throes of massive binary stars," The Astrophysical Journal Letters, vol. 395, p. L83, 1992.

[8] A. Goldstein, P. Veres, E. Burns et al., "An ordinary short gamma-ray burst with extraordinary implications: FermiGBM detection of GRB 170817a," The Astrophysical Journal, vol. 848, no. 2, p. L14, 2017.

[9] B. P. Abbott, R. Abbott, T. D. Abbott et al., "Gravitational waves and gamma-rays from a binary neutron star merger: GW170817 and GRB 170817a," The Astrophysical Journal, vol. 848, no. 2, p. L13, 2017.

[10] F. Pretorius, "Evolution of binary black-hole spacetimes," Physical Review Letters, vol. 95, no. 12, p. 121101, 2005.

[11] M. A. Aloy, H. T. Janka, and E. Müller, "Relativistic outflows from remnants of compact object mergers and their viability for short gamma-ray bursts," Astronomy \& Astrophysics, vol. 436, no. 1, pp. 273-311, 2005.

[12] M. Campanelli, C. O. Lousto, P. Marronetti, and Y. Zlochower, "Accurate evolutions of orbiting black-hole binaries without excision," Physical Review Letters, vol. 96, no. 11, p. 111101, 2006.

[13] J. G. Baker, J. Centrella, D.-I. Choi, M. Koppitz, and J. van Meter, "Gravitational-wave extraction from an inspiraling configuration of merging black holes," Physical Review Letters, vol. 96, no. 11, p. 111102, 2006.

[14] K. Kiuchi, Y. Sekiguchi, K. Kyutoku, M. Shibata, K. Taniguchi, and T. Wada, "High resolution magnetohydrodynamic simulation of black hole-neutron star merger: mass ejection and short gamma ray bursts," Physical Review D, vol. 92, no. 6, article 064034, 2015.

[15] F. Acernese, M. Agathos, K. Agatsuma et al., "Advanced Virgo: a second-generation interferometric gravitational wave detector," Classical and Quantum Gravity, vol. 32, no. 2, p. 024001, 2015.

[16] The LIGO Scientific Collaboration, J. Aasi, B. P. Abbott et al., "Advanced LIGO," Classical and Quantum Gravity, vol. 32, no. 7, article 074001, 2015.

[17] B. P. Abbott, R. Abbott, T. D. Abbott et al., "Observation of gravitational waves from a binary black hole merger," Physical Review Letters, vol. 116, no. 6, article 061102, 2016.

[18] R. Abbott, T. D. Abbott, S. Abraham et al., "Gw190412: observation of a binary-black-hole coalescence with asymmetric masses," Physical Review D, vol. 102, no. 4, article 043015, 2020. 
[19] R. Abbott, T. D. Abbott, S. Abraham et al., "Gw190521: a

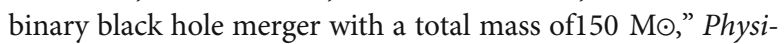
cal Review Letters, vol. 125, no. 10, p. 101102, 2020.

[20] S. V. Dhurandhar, "Data analysis techniques for gravitational wave observations," Pramana, vol. 63, no. 4, pp. 717-730, 2004.

[21] H. E. Hurst, "Long-term storage of reservoirs: an experimental study," Transactions of the American Society of Civil Engineers, vol. 116, pp. 770-799, 1951.

[22] H. E. Hurst, R. P. Black, and Y. M. Simaika, Long-term storage: an experimental study, H. E. Hurst, R. P. Black, and Y. M. Simaika, Eds., Constable London, 1965.

[23] M. Javaherian, H. Safari, N. Dadashi, and M. J. Aschwanden, "Statistical properties of photospheric magnetic elements observed by the helioseismic and magnetic imager onboard the Solar Dynamics Observatory," Solar Physics, vol. 292, no. 11, p. 164, 2017.

[24] C.-K. Peng, S. V. Buldyrev, S. Havlin, M. Simons, H. E. Stanley, and A. L. Goldberger, "Mosaic organization of DNA nucleotides," Physical Review E, vol. 49, no. 2, pp. 1685-1689, 1994.

[25] W. D. Pesnell, "Solar cycle predictions (invited review)," Solar Physics, vol. 281, no. 1, pp. 507-532, 2012.

[26] C. L. Jones, G. T. Lonergan, and D. E. Mainwaring, "Wavelet packet computation of the hurst exponent," Journal of Physics A: Mathematical and General, vol. 29, no. 10, pp. 2509-2527, 1996.

[27] S. Mollaei, A. H. Darooneh, and S. Karimi, "Multi-scale entropy analysis and Hurst exponent," Physica A: Statistical Mechanics and its Applications, vol. 528, p. 121292, 2019.

[28] M. Costa, A. L. Goldberger, and C.-K. Peng, "Multiscale entropy analysis of complex physiologic time series," Physical Review Letters, vol. 89, no. 6, article 068102, 2002.

[29] M. Costa, A. L. Goldberger, and C.-K. Peng, "Multiscale entropy analysis of biological signals," Physical Review E, vol. 71, no. 2, article 021906, 2005.

[30] M. Costa and A. Goldberger, "Generalized multiscale entropy analysis: application to quantifying the complex volatility of human heartbeat time series," Entropy, vol. 17, no. 3, pp. 1197-1203, 2015.

[31] A. Humeau-Heurtier, "The multiscale entropy algorithm and its variants: a review," Entropy, vol. 17, no. 5, pp. 3110-3123, 2015.

[32] B. P. Abbott, R. Abbott, T. D. Abbott et al., "Gwtc-1: a gravitational-wave transient catalog of compact binary mergers observed by LIGO and Virgo during the first and second observing runs," Physical Review X, vol. 9, no. 3, article 031040, 2019.

[33] R. Abbott, T. Abbott, S. Abraham et al., "Open data from the first and second observing runs of Advanced LIGO and Advanced Virgo," SoftwareX, vol. 13, article 100658, 2021. 